19 Revue d'histoire du XIXe siècle

Société d'histoire de la révolution de 1848 et des

révolutions du XIXe siècle

$31 \mid 2005$

La "Société de 48" a cent ans

\title{
Argent, histoire et mémoire
}

Jean-Yves Mollier

URL : http://journals.openedition.org/rh19/959

DOI : $10.4000 /$ rh19.959

ISSN : $1777-5329$

Éditeur

La Société de 1848

Édition imprimée

Date de publication : 1 décembre 2005

ISSN : 1265-1354

Référence électronique

Jean-Yves Mollier, «Argent, histoire et mémoire », Revue d'histoire du XIXe siècle [En ligne], 31 | 2005

mis en ligne le 19 février 2006, consulté le 19 avril 2019. URL : http://journals.openedition.org/

rh19/959 ; DOI : 10.4000/rh19.959

Ce document a été généré automatiquement le 19 avril 2019

Tous droits réservés 


\title{
Argent, histoire et mémoire
}

\author{
Jean-Yves Mollier
}

Dans la trilogie des mémoires ravivées par Jean-Claude Caron, Alain Faure et moi-même, je suis censé parler au nom d'une expérience bien particulière, pour ne pas dire triviale, celle du trésorier de la Société de « 48 » comme nous l'appelions familièrement. Pourtant, si j'ai bien été amené à assurer cette fonction, de 1982 à 1993, c'est d'une expérience de direction collective d'une société savante que j'ai conservé le souvenir. Certes, au départ, les frontières semblaient nettement tracées et les territoires dévolus à leurs titulaires - le secrétariat général à Alain Faure, la direction du Bulletin à Jean-Claude Caron et la trésorerie à moi-même. Mais, dans la pratique, une direction collégiale se mit en place dès 1983-1984, chacun empiétant sur les plates-bandes de son voisin et s'efforçant d'apporter sa contribution au rayonnement de la Société. À cette volonté que je suis apparemment le seul à souligner ici $^{1}$, plusieurs facteurs contribuaient: un phénomène de génération puisque les trois intéressés avaient entre 28 et 35 ans $^{2}$, qu'ils étaient inscrits en thèse de doctorat d'histoire avec le président sortant, Maurice Agulhon, ou son successeur, Philippe Vigier ${ }^{3}$, que leur cœur battait à gauche plutôt qu'à droite et qu'ils avaient retenu du grand ébranlement de Mai-Juin 1968 l'idée que les jeunes avaient un rôle à jouer pour renouveler l'air dans lequel ils respiraient.

2 Fortement appuyés par Rémi Gossez, comme il a déjà été dit, nous souhaitions aider à la renaissance de la Société d'histoire de la révolution de 1848 et des révolutions du XIX ${ }^{\mathrm{e}}$ siècle parce que nous nous reconnaissions peu ou prou dans ses idéaux. Pas plus que la présidence de la Société des Études jaurésiennes ne gêna jamais Madeleine Rebérioux dans son travail d'historienne ${ }^{4}$, pas plus nous n'imaginions que notre vision de la société française et de la nécessité de ses évolutions nous empêcherait de devenir d'authentiques historiens du XIX ${ }^{e}$ siècle ${ }^{5}$. C'est en effet à ce double prisme de notre engagement universitaire et citoyen qu'il convient d'examiner le travail accompli dans les années 1982-1993 au sein du bureau de notre société, ce que confirme d'ailleurs le renouvellement très important du Comité directeur opéré dans les années 1982-1986. Entre ces deux dates, outre les trois secrétaires, avaient été élus Alain Dalotel et JeanClaude Freiermuth, historiens de la Commune qui ne cachaient pas leur sympathie pour l'insurrection de mars 1871, Odile Krakovitch, aux convictions tout aussi fermes, puis, en 
1986, Pierre Lenoël, avocat et historien qui venait renforcer le bureau ${ }^{6}$ et Françoise Tétard, dont Alain Faure rappelle très justement l'importance. Pour être tout à fait complets, il conviendrait de ne pas négliger l'arrivée de Francis Démier et de Stéphane Michaud en 1984, l'un et l'autre partageant à l'évidence nombre des aspirations de leurs collègues. Si l'on ajoute que le collège des vice-présidents comprenait Michelle Perrot, Maurice Agulhon et Henri Dubief et que participaient activement au Comité directeur Robert Brécy, Raymond Huard et Fernand Rude, on aura compris que la Société de 1848 était bien, alors, une association regroupant, sur des bases électives et affectives, des membres aux horizons certes variés mais dont la sensibilité couvrait la totalité de ce qu'on appelle la gauche, de la plus modérée à la plus exaltée.

Le roman des origines

3 Rien ne me prédestinait à devenir trésorier de notre société si ce n'est, peut-être, le fait que, travaillant avec Maurice Agulhon depuis 1979, j'avais été incité par celui-ci à lire les publications qu'elle faisait régulièrement paraître. L'Impossible prison, publiée au Seuil en 1980 et Les Utopismes sociaux chez Sedes, à la fin de l'année suivante, figuraient déjà dans ma bibliothèque quand mon directeur de thèse me suggéra d'assister aux séances du colloque Blanqui, organisé au Centre Malher les $1^{\mathrm{er}}, 2$ et 3 octobre 1981 . De ces trois journées date mon entrée officielle dans le giron quarante-huitard ${ }^{7}$ où devait m'accueillir, voire m'introniser ou m'initier, Rémi Gossez. Apparemment séduit par mes engagements ou ce qu'il croyait en deviner, il me demanda dans les premiers mois de 1982 de me préparer à lui succéder, et donc d'envisager d'être élu, dans un proche avenir, trésorier de la Société de 1848. Ce sera chose officialisée par le Comité directeur du 28 avril $1983{ }^{8}$ mais, dans les faits, je siégeais dans les deux organismes - CD et bureau depuis 1982 et m'étais ainsi peu à peu habitué à l'idée d'être bientôt le financier - terme pompeux - de l'équipe dirigée par Philippe Vigier. À ce stade de la remontée des évocations du passé, il convient d'ailleurs de lui rendre hommage, ainsi qu'à Maurice Agulhon et à Henri Dubief car les uns et les autres étaient parfaitement conscients des ambitions qui animaient leurs jeunes collègues et ils les encourageaient sans ignorer l'ambiguiité de certaines d'entre elles ou le désir des cadets de remplacer un jour les aînés. Toutefois la certitude qui animait Philippe Vigier que notre énergie impulserait un sang neuf à l'organisme qu'il présidait le conduisit à nous accorder toute sa confiance et son appui, décisif à bien des égards ${ }^{9}$.

4 Convaincu, comme l'ensemble du bureau, que les temps étaient advenus d'un renouvellement, et même, peut-être, d'un aggiornamento de la société, Philippe Vigier avait accepté la proposition de Rémi Gossez de remplacer Pierre Ayçoberry, nommé à l'université de Strasbourg, fin 1980, par Alain Faure, devenu en avril 1981 le nouveau secrétaire général. Soucieux, comme il l'explique abondamment dans ce volume, de conférer aux travaux de la société une dimension plus moderne, moins académique, et préférant les journées d'études aux colloques, ce dernier bénéficiait, comme Jean-Claude Caron, élu en même temps que lui, de ses encouragements. Pour ma part, j'ignorais alors pourquoi le trésorier en exercice m'avait pris sous sa protection mais je compris vite, en le côtoyant, que mon parcours personnel y était pour quelque chose et que mes convictions le séduisaient. Très marqué par l'expérience de la Seconde Guerre mondiale et par son séjour en Yougoslavie en 1948, tout autant que par la fidélité au message de la Révolution de 1848 et à l'action de son père dans la société fondée en 1904, Rémi Gossez voulait être certain que la flamme ne serait pas transmise à des enfants prodigues. Cela le conduisit parfois à s'opposer violemment à tel ou tel - Alain Faure aborde cet aspect de la 
vie de la société - mais, avec moi, il ne se départit jamais de son attitude initiale. Il devait me confier un peu plus tard une partie de ses archives, celles qui avaient rapport avec la trésorerie ${ }^{10}$, et, surtout, m'appuyer dans mon effort pour doter l'association de ressources adaptées à ses nouveaux objectifs.

5 Je devais en effet rapidement m'apercevoir que les caisses étaient vides, que plus personne ne payait de cotisation, faute d'appel ou de rappel et que le trésorier n'osait relancer les adhérents dans la mesure où, depuis 1956, la Bibliothèque de 1848 avait cessé de paraître ${ }^{11}$ et où les recueils annuels qui suivirent - du tome XIX au tome XXIV, d'Aspects de la crise en 1956 aux Ouvriers de Paris en $1967^{12}$ - avaient connu une périodicité élastique et où ils s'étaient interrompus après cette date. Offert à tout adhérent à jour de sa cotisation à la veille de 1968 - 15 francs de 1967 valent 15 euros de 2005 - le volume devait durablement endetter son concepteur, Rémi Gossez, dont l'emprunt personnel au Crédit municipal de Paris fut acquitté par lui seul et non par la société. Révélatrice des pratiques et des idéaux des temps héroïques, cette manière de faire risquait de conduire à l'asphyxie si on n'y mettait pas rapidement fin, ce qui explique aussi la volonté du trésorier de confier cette tâche à plus jeune que lui. Comme il me le répéta à plusieurs reprises, il lui répugnait de réclamer des cotisations à des collègues, souvent professeurs ou doyens de leur université - de Jean Bouvier à Jean Vidalenc, en passant par Jacques Droz, Jacques Godechot, Louis Girard ou Albert Soboul, tous présents en certaines occasions au Comité directeur - sans pouvoir leur adresser autre chose qu'une circulaire annuelle. Le compagnonnage de la Société de 1848 avec les Annales historiques de la Révolution française, de 1975 à 1977, et avec Romantisme après cette date, avait été une solution d'attente mais elle ne satisfaisait pas la plupart des adhérents malgré la publication d'études qui firent date, de la première mouture de L'Impossible Prison à 1830 ou Écriture et folie ${ }^{13}$. Manifestement, l'heure était au changement et si la conjoncture politique ne pesait pas directement sur le calendrier de la Société d'histoire de la révolution de 1848, le climat général imposait une mue de l'association.

L'argent nerf de la guerre

6 Sans avoir bien présents à l'esprit tous ces éléments, j'acceptai, quelques semaines après la tenue du colloque organisé à l'occasion du centenaire de la mort d'Auguste Blanqui, de seconder Rémi Gossez et de me plonger dans les délices de la gestion financière d'une société savante. Parcourant les fiches bristol, nominatives, que le trésorier rangeait alors dans une boîte à gâteaux en métal ${ }^{14}$, je m'aperçus très vite que la majorité de ceux qui étaient considérés comme adhérents de la société ne cotisaient plus depuis un grand nombre d'années, ce qui expliquait l'absence quasi totale de fonds propres. Deux décisions furent alors adoptées pour remédier à cette situation, l'augmentation de $25 \%$ de la cotisation, passée de 40 francs en 1981 à 50 francs en 1982 - de 11,70€ à $13 €$ en monnaie constante - puis à 60 francs en $1983-14,30 €$ - et la vente de collections, complètes ou disparates, de la revue de la société. La seconde solution rallia tous les suffrages puisqu'il s'agissait d'enlever des caves de l'appartement du trésorier des séries de publications s'échelonnant de 1904 à 1967 et d'en confier la vente à la librairie Clavreuil, rue Saint-André-des-Arts. Spécialisée depuis sa création, en 1878, dans le livre ancien à caractère historique, cette solide maison parisienne qui entretenait des liens privilégiés avec la Société des Études robespierristes possédait de nombreux clients au Japon et aux États-Unis. Dès 1983, la vente pour 18250 francs - 4361 euros - de collections anciennes rétablissait les finances de la Société de $" 48{ }^{15}$ et permettait de payer rubis sur l'ongle le numéro 1 de la future revue alors à l'étude ${ }^{16}$. 
7 Le second remède suscita davantage de discussions et la hausse des cotisations - 60 francs en 1983 et 1984, 80 francs en 1985 - soit 14,30€ puis $17,80 €$ - ne fut vraiment acceptée par le bureau et le Comité directeur que comme contrepartie de l'édition d'une revue annuelle. S'il est vrai que cette cotisation avait doublé - 40 francs en 1981, 80 francs en 1985 , mais $11,70 €$ puis $17,80 €$ en monnaie constante - l'enjeu était justifié puisque chaque adhérent allait désormais recevoir une revue de 120 pages en lieu et place d'une simple circulaire. Avec une centaine de membres à jour en 1985, un rythme d'adhésions important, 72 en 1985, 62 en 1986, 34 en 1987 et 25 en 1988, la Société d'histoire de la révolution de 1848 dépassait les 200 adhérents à jour de leur cotisation en 1987, les 250 en 1990 et se stabilisait à ce niveau jusqu'à mon départ en $1993{ }^{17}$. La cotisation était passée à 100 francs en $1986-20,40 €$ - puis à 120 francs en $1989-22,30 €$ - mais le volume annuel avait grossi de 136 pages en 1986 à 148 en 1987, 210 en 1988, 220 en 1989 et 216 en 1990 , ce qui correspondait pratiquement à un doublement de la pagination de la revue quand, en euros constants, le prix avait augmenté de $14,30 €$ en 1985 à $22,30 €$ en $1989{ }^{18}$. La dernière hausse de l'adhésion - 150 francs en 1992 ou 25,50€- acheva de stabiliser les finances de la société et si Pierre Lenoël, actif à mes côtés au poste de trésorieradjoint, plus spécialement chargé de la vente des numéros anciens, et moi avions proposé ce dernier ajustement, c'est que les adhérents l'acceptaient, comme l'illustrent la courbe des effectifs et le pic enregistré en 1990-1993, années fastes, même si un certain essoufflement semblait s'annoncer, exigeant d'autres adaptations et d'autres initiatives pour faire connaître l'activité de la société.

Outre les cotisations, la vente des numéros de l'ancienne Bibliothèque de 1848 avait également procuré des ressources relativement importantes à partir de 1984 et un appel aux dons des adhérents apporté des contributions non négligeables ${ }^{19}$, le tout assurant, pour l'essentiel, la publication d'une revue annuelle de qualité et la tenue de colloques nombreux et réguliers à partir de 1983. Si l'argent ne saurait expliquer à lui seul la vitalité d'une organisation, j'avais, pendant toutes ces années, essayé de convaincre le bureau et le Comité directeur qu'il était indispensable au bon fonctionnement de l'association et que l'on ne pouvait tolérer de trop longs écarts dans le paiement des cotisations. En imposant cette règle à tous, quels que soient le grade et la position universitaire de l'intéressé, j'avais apparemment convaincu le plus grand nombre de la justesse de cette attitude. Sans rejeter quiconque et en prenant soin d'adresser plusieurs lettres de rappel avant de radier un adhérent ${ }^{20}$, nous étions parvenus à assainir les finances de la Société de 1848 et à transmettre à nos successeurs - Jean-Luc Mayaud, pour la trésorerie, Jacqueline Lalouette pour le secrétariat et Evelyne Lejeune-Resnick pour les publications - une association en plein renouveau dont Alain Corbin avait pris la direction au début des années 1990. Une page pouvait être tournée mais il serait absurde d'imaginer que j'avais pris seul les décisions concernant la trésorerie comme il serait erroné de penser que Jean-Claude Caron et Alain Faure avaient travaillé isolés dans le secteur qui leur était confié.

Une équipe homogène

9 De ces années de travail à la direction de la Société de « 48 », j’ai en effet conservé le souvenir vivace d'une assez bonne entente avec Alain Faure, passé le premier mouvement d'inquiétude quant à mes engagements qu'il ne partageait pas, et d'une amitié franche avec Jean-Claude Caron. Nous n'hésitions pas à faire précéder ou à prolonger les réunions du Comité directeur de longs moments d'échange et de discussions parfois vives. Ainsi se préparèrent et la gestation de la nouvelle revue et les deux colloques sur Maintien de 
l'ordre et polices en France et en Europe au XIX ${ }^{e}$ siècle, tenu à la Fondation Thiers en décembre 1983 - ce qui souleva quelques réserves de notre part - et sur Répression et prisons politiques en France et en Europe au XIX ${ }^{e}$ siècle, organisé deux ans et demi plus tard, mais à l'université Paris X-Nanterre, celui-là ${ }^{21}$. S'il est certain que le secrétaire général prit la plus large part à la préparation de ces deux rencontres, il le fit en accord avec le bureau, avec Philippe Vigier et Francis Démier avec qui il travaillait à Nanterre, avec Maurice Agulhon qui intervint plus d'une fois sur ces sujets, avec Henri Dubief comme Michelle Perrot et avec Jean-Claude Caron et moi, Pierre Lenoël nous rejoignant en 1986. La publication, en mai 1983, des Mémoires de Ferdinand Gambon ${ }^{22}$ m'avait rendu très sensible aux questions de maintien de l'ordre sur lesquelles je travaillais depuis la préparation de ma première thèse en 1975-1978 ${ }^{23}$. Jean-Claude Caron y était tout aussi attaché puisque "ses » étudiants parisiens des années 1815-1851 avaient eu maille à partir en maintes occasions avec la police ${ }^{24}$. Quant à Alain Faure, son livre, rédigé avec Alain Dalotel et Jean-Claude Freiermuth sur le mouvement des réunions publiques à Paris à la veille de la Commune ${ }^{25}$ témoignait de son intérêt pour ces questions.

De la même manière, mes propositions de hausse des cotisations de la société étaient âprement discutées par Alain Faure, qui craignait qu'elles n'écartent les étudiants et les non-universitaires. Jean-Claude Caron les soutenait parce qu'il voulait doter la Société de 1848 d'une revue mais, là encore, nous eûmes de longs débats, en bureau et hors bureau, en réunion et au téléphone, entre nous et avec les autres membres du Comité directeur. Dans mon souvenir, c'est d'ailleurs Odile Krakovitch qui mit les deux camps d'accord - ceux qui voulaient garder visible la date de 1848 et ceux qui souhaitaient davantage d'amplitude dans la couverture des événements ${ }^{26}$ - en proposant le titre finalement retenu fin 1984 pour la publication: 1848. Révolutions et mutations au XIXe siècle. Il avait l'avantage d'ancrer de plain pied la revue dans le mouvement, de lier le politique au social tout en se voulant à l'écoute des changements. Les premiers sommaires manifestent cet ancrage comme mon souci d'amener à « 48 » les littéraires avec qui Maurice Agulhon, Alain Corbin et moi travaillions à Romantisme et à la Société des Etudes romantiques et dix-neuviémistes.

Dans le tome 2/1986 de 1848. Révolutions et mutations au XIXe siècle, deux articles sur six relevaient de l'histoire littéraire, trois sur sept en 1987 et autant en 1988 - ceux signés Georges Lubin, Bernard Leuilliot, Roger Bellet, Claude Schopp, Francis Ambrière, Louis Le Guillou et Sylvie Chevalley. De même, les comptes rendus de lecture -- dont beaucoup sont signés Jean-Claude Caron et Jean-Yves Mollier - faisaient-ils une large place aux publications littéraires afin de faire venir à la Société ou à la revue des collègues et étudiants qui aimaient l'histoire sans en être des professionnels. De ce point de vue, il fallait ménager ceux qui, à " 48 ", avaient mal vécu le mariage momentané avec Romantisme et ceux qui, dans cette revue amie, trouvaient que l'histoire n'apportait rien à la littérature. Le soutien amical de Stéphane Michaud, bien relayé à la Société des Études romantiques par Max Milner, Pierre Citron, Bernard Leuilliot, Jacques Seebacher et quelques autres, aida à renforcer les liens entre les deux sociétés et les deux revues, ce à quoi Jean-Claude Caron et moi veillions avec attention. Là encore, le soutien de Maurice Agulhon et de Philippe Vigier nous était acquis et l'entrée de Stéphane Michaud au Comité directeur concrétisa cette orientation. Elle fut suivie d'effet, comme on l'a dit, en matière de comptes rendus des publications littéraires et, en octobre 1990, par la tenue, au Musée d'Orsay, du colloque Usages de l'image au XIXe siècle dont Stéphane Michaud fut la cheville ouvrière ${ }^{27}$. Spécialiste de Flora Tristan, professeur de littérature générale et 
comparée, il contribuait à maintenir en notre sein très vivace la flamme romantique, ce qui explique la place consacrée par la revue aux œuvres de Victor Hugo, Félicité de Lamennais, Alexandre Dumas et George Sand comme au théâtre de cette époque.

À côté de ces collaborations étroites mais qui relèvent du travail de préparation théorique des sommaires de 1848. Révolutions et mutations au XIXe siècle, il existe tout un arrière-plan plus prosaïque qui ne saurait être caché. Pour mettre sous pli la revue, il fallait en effet aller en chercher les numéros à l'imprimerie où elle était confectionnée, la ramener Villa Ledoux, à Nogent-sur-Marne, où résidait Jean-Claude Caron, acheter enveloppes et timbres, la mettre sous pli puis se rendre à la poste pour négocier l'expédition dans les meilleurs délais. Nous nous retrouvions donc, Alain Faure, Jean-Claude Caron et moi pour effectuer ces tâches pratiques et, bien entendu, parler de l'avenir des futurs numéros, des colloques ou des journées d'études, de l'avancement de nos thèses, de nos espoirs et de nos déceptions. Plus âgé que mes deux complices, je devais, le premier, intégrer l'université, à Nanterre précisément, ce qui me donna l'occasion de rencontrer plus fréquemment Alain Faure et de travailler en étroite collaboration avec Philippe Vigier ${ }^{28}$. Paradoxalement, cela ne se traduisit pas par un rapprochement avec le secrétaire général, hostile, il l'a dit, à l'organisation de gros colloques, et de plus en plus inquiet devant le mouvement de hausse des cotisations qui risquait, selon lui, d'écarter certains historiens amateurs de notre société.

Pourtant le succès rencontré par la publication de L'Impossible prison puis des Utopismes sociaux nous avait convaincus, Jean-Claude Caron et moi, qu'une société telle que " 1848 » se devait de faire connaître régulièrement ses grands thèmes d'études et que cela passait par la préparation de grands colloques internationaux permettant la confrontation des points de vue. Chargés avec Philippe Vigier de négocier auprès des éditeurs d'alors, CDU et SEDES, les actes de la rencontre de 1981, Blanqui et les blanquistes, nous avions dû attendre 1986 pour voir paraître le volume et nous avions compris que cette maison d'édition ne nous accompagnerait plus dans nos efforts pour porter nos travaux à la connaissance du public. Cela facilita notre décision de faire appel à deux jeunes docteurs en histoire, tous deux spécialistes d'histoire orale, Claire Reverchon et son mari, Pierre Gaudin, qui venaient de créer la maison Créaphis. Participant activement aux activités de la Société de « 48 », intervenants lors du colloque " Maintien de l'ordre », ils allaient se voir confier la fabrication et la diffusion des prochains volumes d'actes - de Maintien de l'ordre et polices en France et en Europe au XIX siècle, publié en 1986, jusqu'au très récent Comment meurt une république. Autour du 2 Décembre 1851, paru en 2004, ce qui prouve à quel point leur intégration dans la société, et bientôt au Comité directeur, avait été bénéfique. Elle démontre également le caractère collectif du travail effectué par la jeune génération pendant toutes ces années. Avec Pierre Lenoël, Françoise Tétard et d'autres, des prolongements presque naturels à la diffusion de nos recherches avaient été trouvés puisqu'ils s'étaient lancés dans la bataille des radios dites «libres » après 1981. Tant à Radio Montmartre qu'à Radio Aligre ensuite, la Société d'histoire de la révolution de 1848 rencontra de ce fait des médiateurs amicaux qui relayèrent auprès des auditeurs ses travaux et ses publications, ce qui contribua indiscutablement à son rayonnement.

Le passage de témoin

14 Alain Faure a expliqué son retrait progressif du bureau puis du Comité directeur de " 48 » en insistant sur ses désaccords avec les nouvelles orientations en matière de colloques. Pour ma part, j'étais et je demeure persuadé qu'il n'existait aucune raison pour que les journées d'études se révèlent plus productives que les rencontres de plus grande 
ampleur. Persuadé que le travail des universitaires auprès de leurs étudiants avancés, dès la maitrise, était indispensable pour faire connaître la Société, sa revue et ses publications, j'avais essayé de sensibiliser tous les centres d'archives départementales et toutes les bibliothèques universitaires à nos activités. Le résultat avait été excellent dans certaines régions, médiocre ou nul ailleurs, ce qui confirmait la nécessité des relais régionaux et internationaux, tâche assumée bientôt par nos correspondants ${ }^{29}$. Des bulletins d'adhésion étaient régulièrement confiés aux membres du Comité directeur et aux adhérents lors des assemblées générales mais cela ne suffisait pas et le cap des 250 cotisants, nous l'avons dit, fut à la fois un heureux résultat en même temps qu'une sorte de barrière que nous ne parvînmes pas à dépasser. Sans doute cela me convainquit-il qu'il était temps de faire appel à d'autres énergies, capables à leur tour, comme nous l'avions fait à partir de 1981-1982, de proposer des solutions adaptées aux besoins des années 1990-1995, le deuxième septennat de François Mitterrand étant, à l'évidence, différent du premier et l'époque ayant beaucoup changé.

15 À nos débuts, préparant à la fois l'édition des Mémoires de Ferdinand Gambon, sept ans enfermé à la prison de Belle-Ile-en-Mer, j'avais songé à profiter de l'arrivée de Robert Badinter au ministère de la Justice, pour essayer de faire ériger un musée du souvenir dans ce haut lieu de la détention et de la déportation des politiques. Avec Françoise Tétard, une nouvelle fois, nous avions sondé tant l'administration pénitentiaire que les archives départementales du Morbihan. Le directeur de ce dépôt m'avait laissé bien peu d'espoir compte tenu de son intime connaissance des sensibilités locales. De fait, après quelques séjours sur place, à la Citadelle devenue un Musée privé et sur les glacis, là où avaient été construits les baraquements qui servirent de cellules aux transportés de Juin 1848 puis de prison politique de 1849 à 1857 et, de nouveau, de 1871 à $1880^{30}$, je compris à quel point il avait raison. Les incidents survenus à l'été 1983 entre des jeunes venus de cités sensibles de la Région parisienne et encadrés par des policiers volontaires et les vacanciers qui ne supportaient pas leur présence achevèrent de ruiner nos espoirs et ceux de la Société de 1848 qui aurait dû initier ce que l'on n'appelait pas encore un « devoir de mémoire ${ }^{31}$.

16 Toutefois, l'une des retombées de ces tentatives devait se révéler beaucoup plus heureuse. Sollicitée par Robert Badinter pour ouvrir une réflexion sur les questions posées par la prison à la démocratie, Michelle Perrot, toujours vice-présidente de la Société d'histoire de la révolution de 1848, accepta ce projet avec enthousiasme. À l'automne 1986, s'ouvrait ainsi, à l'École des Hautes Études en Sciences Sociales, un séminaire sur la prison républicaine dirigé par Michelle Perrot et Robert Badinter. Dominique Kalifa, Martine Kaluszynski, Odile Krakovitch, Michel Pierre, Françoise Tétard et moi y participâmes, et si nos interventions ne furent finalement pas publiées dans un volume collectif, comme l'aurait souhaité Michelle Perrot, du moins la publication en 1992 par Robert Badinter de La Prison républicaine (1871-1914) ${ }^{32}$ permit-elle de faire connaitre les résultats de nos recherches, de nos débats et de nos réflexions sur l'avenir. En engageant nombre de ses étudiants sur ce terrain, celle qui avait ouvert le dialogue avec Michel Foucault, au moment du dossier dont sortit L'Impossible Prison, prouvait ainsi la vitalité de la société savante à laquelle elle appartenait et sa capacité à s'emparer des questions que posait la société aux historiens.

17 À l'orée des années 1990-2000, des débats nouveaux se faisaient jour, le militantisme reculait, les grandes idéologies qui avaient brûlé de leur rougeoyante incandescence vingt-cinq ans plus tôt s'estompaient. Certains annonceraient bientôt la fin de l'histoire 
mais, au-delà de cette conjoncture que l'on ne peut toutefois pas ignorer, des raisons plus triviales ou plus prosaïques allaient entraîner des changements à la direction de " 48 ". Avec Alain Corbin, Jacqueline Lalouette, Jean-Luc Mayaud et Evelyne Lejeune-Resnick, une autre équipe se mettait progressivement en place en 1991 et, fin 1992, je fis connaître mon intention de suivre Jean-Claude Caron et Alain Faure, remplacés dans leurs mandats un an avant moi. Jean-Luc Mayaud devait me succéder en 1993, faire passer la société et la trésorerie à l'heure de l'informatique, ce qui était devenu une nécessité. Pour ma part, nommé professeur d'histoire contemporaine à l'université de Versailles Saint-Quentinen-Yvelines à l'automne 1992, et engagé, avec Pascal Ory, dans la création du Centre d'histoire culturelle des sociétés contemporaines, je n'avais plus le temps de me consacrer activement aux travaux de notre société. Encore très présent en 1989 dans la préparation du numéro sur Le devenir des révolutions et, en 1990, dans le débat sur Le XIXe siècle en question, je considérais qu'il fallait laisser une autre équipe entourer un autre président, ce qui m'amena à me retirer du bureau à l'Assemblée générale du printemps 1993, heureux du travail accompli, de l'expérience enrichissante que je venais de traverser et de ma certitude que notre société avait encore sa place dans le paysage scientifique.

Avec Odile Krakovitch et Raymond Huard comme vice-présidents, l'équipe de direction était assurée d'une réelle continuité et il allait lui appartenir de trancher bientôt entre une fidélité un peu encombrante aux stricts idéaux de 1904 et une adaptation aux exigences de la fin du $\mathrm{XX}^{\mathrm{e}}$ siècle. Il ne s'agissait ni de rompre totalement avec certains engagements ni de méconnaître les aspirations des nouveaux adhérents. Pour ces raisons et quelques autres qui ont été avancées par Alain Faure et Jean-Claude Caron, je partais sans amertume, convaincu que ce fut une chance exceptionnelle pour le jeune historien que j'étais à l'aube des années 1980 d'avoir pu côtoyer la génération des Jacques Droz, Ernest Labrousse et Albert Soboul, puis la suivante, de Maurice Agulhon à Philippe Vigier en passant par Michelle Perrot, et d'avoir essayé d'apprendre auprès d'eux le métier qui était devenu le mien. À leurs côtés, j'avais pu rencontrer des dizaines d'autres spécialistes de cette discipline, français ou étrangers, provinciaux ou parisiens, échanger avec beaucoup d'entre eux une correspondance passionnante et élargir ainsi mon horizon. On comprendra donc aisément pourquoi, au moment de conclure cette évocation du passé, je songe à Rémi Gossez et à son inébranlable confiance dans son successeur.

\section{NOTES}

1.. Ni Jean-Claude Caron ni Alain Faure ne l'évoquent dans leurs contributions.

2.. Je suis né en 1947, Alain Faure en 1949 et Jean-Claude Caron en 1955.

3.. Jean-Claude Caron préparait une thèse de doctorat nouveau régime avec Maurice Agulhon, Alain Faure et moi une thèse de doctorat d'État, lui, avec Philippe Vigier, moi avec Maurice Agulhon. Jean-Claude Caron soutiendra sa thèse en 1989, avec Maurice Agulhon, Alain Corbin et Philippe Vigier dans son jury, moi en 1986, avec Philippe Vigier pour président. J'étais déjà titulaire d'un doctorat de $3^{\circ} \mathrm{cycle}$ depuis 1978 . Jean-Claude 
Caron et moi enseignions alors dans le secondaire tandis qu'Alain Faure occupait un poste d'IATOS au département d'histoire de l'université Paris X-Nanterre.

4.. Voir « Hommage à Madeleine Rebérioux », dans Cahiers Jaurès n 174, oct-déc. 2004, et Maurice AGULHON, Histoire et politique à gauche, Paris, Perrin, 2005, 162 p.

5.. Il va de soi que si nos sensibilités nous situaient à gauche de l'échiquier politique, nos différences d'appréciation sur le système des partis et des mouvements étaient réelles.

6.. Il allait y assumer le poste de trésorier-adjoint jusqu'à mon départ en 1993 et y accomplir sa part de travail.

7.. Grâce à l'obligeance de M. Agulhon, j'avais pu obtenir une décharge de cours pour ce colloque, pratique alors peu en vigueur dans l'enseignement secondaire.

8. Il va de soi que j'ai utilisé, outre ma mémoire, toujours faillible, mes archives et mes agendas pour rédiger cet article.

9.. Il veilla sur la carrière universitaire de nombre d'entre nous et, pour ma part, je n'ai jamais oublié tout ce que je luis dois, y compris mon élection comme Maître de Conférences en histoire contemporaine à l'université Paris X-Nanterre en septembre 1988.

10.. Je les ai intégralement confiées à mon successeur, Jean-Luc Mayaud, en 1993.

11.. Rémi GOSSEZ, « La Société de '1848’ (1945-1980) », dans 1848. Révolutions et mutations au XIX siècle, n² 2/1986, pp. 17-18.

12.. Ibidem.

13. Dans AHRF et Romantisme ; voir Rémi GOSSEZ, art. cité, pp. 19-21.

14.. Je l'ai scrupuleusement transmise à Jean-Luc Mayaud en 1993.

15.. Cette somme équivalait pratiquement à l'encaisse du 1/01/1983 - 18562 francs.

16. Le $n^{\circ}$ de 1985 coûta 18874,50 francs pour les 300 premiers exemplaires et 2354 francs pour les 50 exemplaires commandés après épuisement du tirage initial.

17.. 256 adhérents à jour en 1991, 248 en 1993 et 215 en 1994 selon mes archives.

18. Nous avons utilisé le déflateur de l'INSEE pour tenir compte de la hausse des prix et de l'inflation pendant toutes ces années.

19.. On ne citera ici que deux des donateurs les plus constants, les regrettés Henri Dubief et Edgar Newman.

20.. En fait, il ne recevait plus la revue, ce qui était la seule sanction de sa suspension des cotisations.

21.. Tous les deux ont été publiés par Créaphis, le premier en 1986, le second en 1990.

22. Dans les bagnes de Napoléon III. Mémoires de Charles-Ferdinand Gambon, Paris, Presses universitaires de France, 1983, 296 p.

23.. Jean-Yves MOLLIER, Noël Parfait (1813-1896). Biographie littéraire et historique, thèse de doctorat de $3^{\circ}$ cycle de littérature française, dir. Pierre Citron, université Paris 3, 1978, 2 vol.

24.. Jean-Claude CARON, Générations romantiques. Les étudiants de Paris et le Quartier Latin (1815-1851), Paris, Armand Colin, 1991, 435 p.

25. Alain DALOTEL, Alain FAURE et Jean-Claude FREIERMUTH, Aux origines de la Commune. Le mouvement des réunions publiques à Paris. 1868-1870, Paris, Maspero, 1980, 375 p.

26. Voir l'article de Jean-Claude Caron sur ce point.

27.. Usages de l'image au XIXe siècle, Stéphane MICHAUD, Jean-Yves MOLLIER et Nicole SAVY [dir.], Paris, Créaphis, 1992, 260 p.

28. J'avais soutenu mon doctorat d'État en histoire en 1986 et avais été recruté en septembre 1988 à l'université de Paris X-Nanterre. 
29.. Ils apparaissent ès qualités au sommaire de la revue dès le $n^{\circ} 3$ en 1987, année où Jean-Claude Caron, jusqu'ici dénommé « secrétaire de rédaction » ( $n^{\circ} 1$ et 2$)$, devient « secrétaire aux publications », ce qui traduit bien l'évolution et la professionnalisation de 1848.

30.. Voir mes deux communications aux colloques Maintien de l'ordre et Répression et prisons politiques sur ce point.

31.. Présent en août 1983 à Belle-Ile, j'ai conservé un souvenir précis de l'exploitation tendancieuse de ces incidents par les médias nationaux. D'un certain point de vue, l'échec de cette expérience de vacances à la mer pour des jeunes venus des cités défavorisées annonce les changements en cours fin 1983 - début 1984 à l'échelle du pays.

32. Robert BADINTER, La prison républicaine (1871-1914), Paris, Librairie Arthème Fayard, 1992,429 p.

\section{RÉSUMÉS}

Cet article constitue à la fois la remémoration d'une expérience personnelle, celle du trésorier de la «Société d'histoire de la révolution de 1848 » en poste de 1982 à 1993, et une tentative de reconstitution d'un double engagement, universitaire et citoyen, au service d'un idéal militant. Après avoir rappelé son effort pour assainir les finances de l'association et la doter de fonds lui permettant d'assumer, dès 1985, la publication d'une nouvelle revue annuelle, 1848. Révolutions et mutations au XIX ${ }^{e}$ siècle, Jean-Yves Mollier insiste sur le caractère collectif de cet aggiornamento d'une société savante au début des années 1980. Avec Jean-Claude Caron et Alain Faure, mais également avec les membres du bureau et du Comité directeur de la société puis du Conseil d'administration, un important travail fut accompli dont témoignent tant les sommaires de la revue que les volumes issus des nombreux colloques tenus à cette époque. Avec plus de 250 adhérents réellement à jour de leur cotisation au seuil des années 1990, une publication annuelle de qualité et des rencontres internationales en perspective, la Société de « 48 » pouvait considérer que son avenir était assuré, d'où le passage de témoin d'une équipe, celle de Philippe Vigier, à une autre, celle d'Alain Corbin.

Money, history and memory

This article is both the recollection of a personal experience - that of the treasurer of the «Société d'histoire de la révolution de 1848 » from 1982 to 1993 - and an attempt to reconstitute a double commitment, as an academic and as a citizen, so as to serve a certain ideal. Jean-Yves Mollier begins by explaining the efforts he made to stabilize the finances of the association in order to enable the publication of an annual review 1848, Révolutions et mutations au XIXe siècle. He insists on the collective effort that was undertaken for the success of this aggiornamento of a learned society at the beginning of the 1980's. With Jean-Claude Caron and Alain Faure, but also with the members of the board of the society, he centred its efforts around the publication of the review and the proceedings of the numerous conferences organised at that time. With more than 250 members - having really paid their fees on the eve of the 1990's - the Société of « 48 ", thanks to annual publication of good quality and international meetings planned for the years to come, could consider that its future seemed assured. A new team could succeed Philippe Vigier's: Alain Corbin's. 


\section{AUTEUR}

\section{JEAN-YVES MOLLIER}

Professeur d'histoire contemporaine à l'université de Versailles Saint-Quentin-enYvelines 